\title{
A Note on Gaussian Twin Primes
}

\section{By Daniel Shanks}

If $m^{2}+1$ is a prime, then $m+i$ is a Gaussian prime and conversely. If $(n-1)^{2}+1$ and $(n+1)^{2}+1$ are both prime, then $n-1+i$ and $n+1+i$ form a pair of Gaussian twin primes [1, p. 82]. This is the case for $n=3,5,15$, $25,55, \cdots, 184705,184745,184755, \cdots$; the corresponding (rational) primes being 5 and 17 for $n=3$, and 34134040517 and 34134779537 for $n=184755$. Let $g(N)$ be the number of such pairs for $4 \leqq n+1 \leqq N$.

Similarly, let $z(N)$ be the number of pairs of rational twin primes, $n-1$ and $n+1,($ such as $n=4,6,12,18, \cdots)$, for $5 \leqq n+1 \leqq N$. Hardy and Littlewood [2] conjectured that

$$
z(N) \sim 1.32032 \int_{2}^{N} \frac{d n}{(\log n)^{2}}
$$

where

$$
1.32032 \cdots=2 \prod_{p=3}^{\infty}\left(1-\frac{1}{(p-1)^{2}}\right)
$$

the product being taken over all odd primes.

By the use of a sieve argument very similar to that recently presented [3] in support of another Hardy-Littlewood conjecture, the following asymptotic relation was obtained:

$$
g(N) \sim 0.36932 z(N)
$$

where

$$
0.36932 \cdots=\frac{1}{4} \prod_{p=3}^{\infty}\left[1-2\left(\frac{-1}{p}\right)(p-2)^{-1}\right] .
$$

Here $(-1 / p)$ is the Legendre symbol. Assuming the truth of both (3) and (1), we have

$$
g(N) \sim 0.48762 \int_{2}^{. v} \frac{d n}{(\log n)^{2}}
$$

We may compute the constant in (5), and therefore also that in (4), from

$$
0.48762 \cdots=\frac{\pi^{2}}{8} \prod_{p=4 m+1}\left(1-\frac{t}{p}\right)\left(\frac{p+1}{p-1}\right)^{2},
$$

the product being taken over all primes of the form $4 m+1$. The evaluation of the right side of (6) is facilitated by a transformation similar to that previously used [3] in computing the Hardy-Littlewood constants, $h_{a}$.

The number of Gaussian twin pairs, $g(N)$, was determined for $N=$ $500(500) 185000$, by counting these pairs in a recently computed table $[1$, p. 81$]$ of the greatest prime factor of $n^{2}+1$ for $n=1(1) 185000$. A short summary is shown in Table 1 together with a comparison of $g(N)$ and

Received December 31, 1959. 
TABLE 1

Gaussian and Rational Twin Primes

\begin{tabular}{|c|c|c|c|c|c|c|}
\hline$N$ & $g\left(N^{\top}\right)$ & $\bar{g}(x)$ & $\boldsymbol{g} / \overline{\mathbf{g}}$ & $z(N)$ & $\Xi(N)$ & $=\sqrt{2}$ \\
\hline 10000 & 76 & 79.1 & 0.961 & 205 & 214.2 & 0.957 \\
\hline 20000 & 127 & 132.1 & 0.961 & 342 & 357.8 & 0.956 \\
\hline 30000 & 180 & 179.8 & 1.001 & 467 & 486.7 & 0.959 \\
\hline 40000 & 234 & 224.3 & 1.043 & 591 & 607.4 & 0.973 \\
\hline 50000 & 276 & 266.8 & 1.034 & 705 & 722.5 & 0.976 \\
\hline 60000 & 321 & 307.8 & 1.043 & 811 & 833.4 & 0.973 \\
\hline 70000 & 361 & 347.5 & 1.039 & 905 & 940.9 & 0.962 \\
\hline 80000 & 403 & 386.2 & 1.044 & 1007 & 1045.7 & 0.963 \\
\hline 90000 & 437 & 424.0 & 1.031 & 1116 & 1148.2 & 0.972 \\
\hline 100000 & 463 & 461.2 & 1.004 & 1224 & 1248.7 & 0.980 \\
\hline 110000 & 502 & 497.7 & 1.009 & & & \\
\hline 120000 & $5: 32$ & 533.6 & 0.997 & & & \\
\hline 130000 & 568 & 569.0 & 0.998 & & & \\
\hline 140000 & 598 & 603.9 & 0.990 & & & \\
\hline 150000 & 629 & 638.4- & 0.985 & & & \\
\hline 160000 & 660 & 672.6 & 0.981 & & & \\
\hline 170000 & 696 & 706.4 & 0.985 & & & \\
\hline 180000 & 734 & 739.8 & 0.992 & & & \\
\hline 185000 & 762 & 756.4 & 1.007 & & & \\
\hline
\end{tabular}

$$
g(N)=0.48762 \int_{2}^{N} \frac{d n}{(\log n)^{2}} .
$$

Also shown are Glaisher's counts [4] of $z(N)$ to $N=10^{5}$ and

$$
\bar{z}(N)=1.32032 \int_{2}^{N} \frac{d n}{(\log n)^{2}} .
$$

In this range of $N$ the deviations from unity of $g(N) / \tilde{g}(N)$ and $z(N) / \bar{z}(N)$ are about equal in magnitude, [5].

The slow oscillations of $g(N) / \bar{g}(N)$ around one have two significant consequences.

1. They make improbable any value of the constant in (5) which differs more than slightly from the theoretical value, (6).

2. They make possible a sensitive test for the correctness of the function of $N$ assigned to the proposed asymptote, $\int_{2}^{N} d n /(\log n)^{2}$. For since $|g(N)-\bar{g}(N)| \ll$ $\vec{g}(N)$, even small functional modifications in $\bar{g}(N)$ would greatly alter the phase, frequency, and amplitude of the corresponding oscillations of $g(N) / \tilde{g}(N)$ around one. Now consider $P(N)$, the total number of Gaussian primes of the form $m+i$, $[1$, see Table on p. $78 ;$ p. 81$]$. The corresponding Hardy-Littlewood conjecture reads

$$
P(N) \sim \bar{P}(N)=0.68641 \int_{2}^{N} d n / \log n,
$$

and similar remarks are applicable to the function $\bar{P}(N)$ and to any oscillations of $P(N) / \bar{P}(N)$. But if $(9)$ and $(5)$ are both valid, we must expect that any slow oscillations of $g(N) / \bar{g}(N)$ and $P(N) / \bar{P}(N)$ will agree in phase and frequency. For, where 


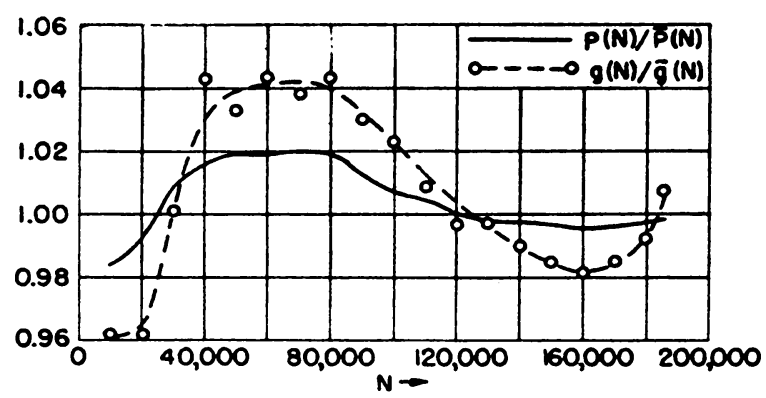

FIG. 1-Comparison of $g(N) / \bar{g}(N)$ with $P(N) / \bar{P}(N)$.

there is an excess of primes, there should generally also be an excess of twins, and if the oscillations are slow, then any complicating higher frequency fluctuations in the local density will largely disappear by integration. In Figure 1 we compare graphs of $g(N) / \bar{g}(N)$ and $P(N) / \bar{P}(N)$ for $N$ to 185000 . Very close agreement is seen in the phase and frequency of the slow oscillations. Since such an agreement would seem improbable if either or both of (5) and (9) were false, it may be regarded as providing further evidence in their favor.

The difficulties that stand in the way of a proof of (3), (assuming it to be true) are similar to those previously discussed for other problems [3]. Thus it is unlikely that (3) will be proven without a simultaneous solution of the long outstanding Goldbach, twin prime, and $n^{2}+1$ prime problems.

In conclusion it should be noted that the Gaussian twins on the line $n+i$ are by no means the only "twins" in the Gauss plane. On the line $n+2 i$, for instance, we not only have twins, $n=(179983,179985)$, and triplets, $n=(423,425,427)$, but even one octuplet, $n=(-7,-5,-3,-1,+1,+3,+5,+7)$.

Applied Mathematics Laboratory

David Tayior Model Basin

Washington 7, District of Columbia

1. DANIEL SHANKS, "A sieve method for factoring numbers of the form $n^{2}+1$." $Y T \cdot A \bar{C}$, v. 13,1959 , p. $78-86$

2. G. H. HARDY \& J. E. LitTlewond, "Partitio numerorum III: On the expression of a number as a sum of primes," Acta. Wath., v. 4,1923, p. 42.

3. Da NIEL SHANKS, "On the conjecture of Hardy and Littlewood concerning the number of primes of the form $n^{2}+a$," Notices, Amer. Math. Soc., v. 6, 1959, p. 417. Abstract 559-52. A forthcoming paper with the same title will give an expanded version of this report. $28-33$

4. J. W. L. Glaisher, "An enumeration of prime-pairs," Messenger Math., v. 8, 1sis. p.

5. The empirical evidence for (1) is much more extensive. D. H. Lehmer has computed $z(N)=183728, \bar{z}(N)=183582$, and $z / z(N)=1.0008$ for $N=37 \cdot 10^{6}$. See the review, UNIT 3 , of D. H. LEHMER, "Tables concerning the distribution of primes up to 37 million." .IT $A C$, v. 13,1959 , p. 56 . 